\title{
Fermi-level effects on the electronic structure and magnetic couplings in (Ga,Mn)N
}

\author{
P. Bogusławski ${ }^{1,2}$ and J. Bernholc ${ }^{2}$ \\ ${ }^{1}$ Institute of Physic PAS, 02668 Warszawa, Poland \\ ${ }^{2}$ Center for High Performance Simulation and Department of Physics, North Carolina State University, \\ Raleigh, North Carolina 27695, USA \\ (Received 11 March 2005; revised manuscript received 16 May 2005; published 20 September 2005)
}

\begin{abstract}
Electronic structure of $\mathrm{Mn}$ ions and the magnetic $\mathrm{Mn}-\mathrm{Mn}$ coupling in $(\mathrm{Ga}, \mathrm{Mn}) \mathrm{N}$ are investigated within density functional theory. The properties of $(\mathrm{Ga}, \mathrm{Mn}) \mathrm{N}$ are found to critically depend on the position of the Fermi level, which alters the charge state of Mn ions and induces substantial lattice relaxation. The exchange splittings of Mn $d$ levels decrease threefold with the decreasing Fermi level, with the magnetic couplings being similarly affected. The results are in excellent agreement with experiment for low Mn concentration and highlight the role of inhomogeneities at the high concentration limit.
\end{abstract}

DOI: 10.1103/PhysRevB.72.115208

PACS number(s): 75.50.Pp, 75.30.Hx, 71.15.Mb

\section{INTRODUCTION}

In recent years, the prospect of spintronic applications ${ }^{1-4}$ motivated a considerable effort devoted to search for semiconductors with ferromagnetism controlled by band carriers and Curie temperatures exceeding the room temperature. Particular attention was focused on $(\mathrm{Ga}, \mathrm{Mn}) \mathrm{N}$, which was predicted to exhibit the highest Curie temperature $T_{\mathrm{C}}$ in the III-V family. ${ }^{4}$ Recent experiments ${ }^{5-8}$ have produced a consistent picture of the electronic structure of an isolated Mn atom in $\mathrm{GaN}$, according to which $d(\mathrm{Mn})$ states are located in the lower part of the $\mathrm{GaN}$ band gap. In this situation the Zener model ${ }^{4}$ of magnetism mediated by band carriers is not applicable. On the other hand, experimental investigations of the magnetic properties of $(\mathrm{Ga}, \mathrm{Mn}) \mathrm{N}$ have provided ambiguous results. In $n$-type samples with low Mn content, the observed Mn-Mn coupling is antiferromagnetic (AFM) ${ }^{9-11}$ For higher Mn contents, ferromagnetic (FM) coupling with $T_{\mathrm{C}}$ exceeding $300 \mathrm{~K}$ has been observed in $n$-type and/or insulating, ${ }^{10,12-17}$ as well as $p$-type ${ }^{18,19}$ samples. A possible explanation of these complex magnetic properties is that the $\mathrm{Mn}$ distribution is not random and the morphology of samples with high Mn content is highly nonideal due to, e.g., very high concentrations of point defects [e.g., interstitial $\mathrm{Mn}$, as in the case of as-grown ( $\mathrm{Ga}, \mathrm{Mn}) \mathrm{As}$ (Ref. 20)], or inclusions of magnetic phases due to segregation effects in $(\mathrm{Ga}, \mathrm{Mn}) \mathrm{N}$. In fact, inclusions of antiferromagnetic $\mathrm{GaMn}_{3} \mathrm{~N}_{4},{ }^{16,18}$ ferrimagnetic $\mathrm{Mn}_{4} \mathrm{~N}$, or other $\mathrm{Mn}_{x} \mathrm{~N}_{y}$ phases ${ }^{21}$ have been experimentally detected. Typically only a small fraction, about $1-10 \%$ of Mn spins, participates in ferromagnetism, and ferro- and paramagnetism coexist.

In this situation it is important to recognize which of the observed effects are genuine properties of bulk homogeneous $(\mathrm{Ga}, \mathrm{Mn}) \mathrm{N}$. To this end we extend previous theoretical studies $^{22-29}$ of ideal $(\mathrm{Ga}, \mathrm{Mn}) \mathrm{N}$ and investigate its properties as a function of the Fermi energy $E_{\mathrm{F}}$, i.e., of the charge state of Mn. It turns out that $E_{\mathrm{F}}$ affects the positions of the Mninduced levels and the sign of the Mn-Mn coupling. Moreover, we demonstrate the importance of charge-dependent lattice relaxations around $\mathrm{Mn}$ ions. The results provide a consistent interpretation of a number of experimental data, and indicate which effects should be due to nonuniform distribution of $\mathrm{Mn}$ in actual samples.

\section{METHODOLOGY}

The calculations are performed within the local spin density approximation (LSDA), using the code of Ref. 30. We consider the wurtzite phase of $\mathrm{GaN}$ and use a large unit cell with 72 atoms. Thus, the substitution of one Ga atom by $\mathrm{Mn}$ corresponds to $2.8 \% \mathrm{Mn}$ concentration, etc. Ultrasoft pseudopotentials are used, which enables a relatively low plane wave cutoff of $35 \mathrm{Ry}$, and the Brillouin zone summations are performed using the Monkhorst-Pack $2 \times 2 \times 2$ $k$-point grid in the folded Brillouin zone, which gives convergent results. The positions of all atoms in the unit cell are allowed to relax. In calculations for charged states of $\mathrm{Mn}$ ions, a neutralizing homogeneous background is used. ${ }^{31} \mathrm{We}$ note that LSDA and the generalized gradient approximation give very similar results for $\mathrm{Mn}$ in both GaAs and $\mathrm{GaN} .^{22,23}$ The effects of the electron self-interaction may be taken into account within the LDA $+U$ scheme, which has been used for both $(\mathrm{Ga}, \mathrm{Mn}) \mathrm{As}$ and $(\mathrm{Ga}, \mathrm{Mn}) \mathrm{N} .{ }^{22-24}$ From Refs. $22-24$ it follows that the inclusion of the on-site interaction increases the delocalization of the hole wave function (which increases $T_{\mathrm{C}}$ ) and decreases the $p-d$ coupling (which decreases $T_{\mathrm{C}}$ ). The value of $T_{\mathrm{C}}$ obtained within LSDA and $\mathrm{LDA}+U$ schemes is similar. ${ }^{24(a)}$ The results do not unambiguously show the superiority of either of these schemes in reproducing the experimental data even in the case of the better understood $(\mathrm{Ga}, \mathrm{Mn})$ As.

\section{ELECTRONIC STRUCTURE OF Mn IN GaN AND ATOMIC RELAXATIONS}

The calculated electronic structure of $(\mathrm{Ga}, \mathrm{Mn}) \mathrm{N}$ is schematically shown in Fig. 1 for several charge states of Mn. The states derived from the five spin-up $d(\mathrm{Mn})$ orbitals are in the lower half of the band gap; they are antibonding combinations of the $d(\mathrm{Mn})$ states with the four dangling bonds of the nearest $\mathrm{N}$ neighbors. In the case of an isolated $\mathrm{Mn}$ impurity, the crystal field of the wurtzite lattice splits the fivefold 


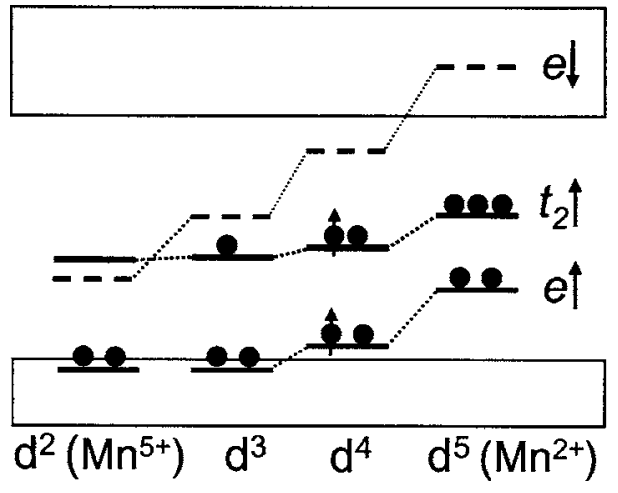

FIG. 1. Energy levels of $\mathrm{Mn}$ in $\mathrm{GaN}$ for charge states ranging from $d^{5}$ to $d^{2}$. Full circles represent electrons. For $d^{2}$ and $d^{3}$ configurations, the $e \uparrow$ level is a resonance within the valence band. The position of the $e \downarrow$ doublet is shown by the dashed line; this level is always empty. For the $d^{5}$ configuration, $e \downarrow$ is a resonance within the conduction band.

degenerate $d$ shell into a doublet $e$ and a pseudotriplet $t_{2}$ separated by the crystal field energy $\Delta_{\text {cr }}$ of about $1.2 \mathrm{eV}$. In $\mathrm{GaN}$ with the zinc-blende structure, the $t_{2}$ state is a triplet. In wurtzite $\mathrm{GaN}$, the $t_{2}$ state is further split into a doublet and a singlet with a small energy difference of about $0.1 \mathrm{eV}$, which is represented by thick lines in Fig. 1. This small splitting is largely independent of the $\mathrm{Mn}$ charge state. $\mathrm{In}_{\mathrm{Ga}_{1-x}} \mathrm{Mn}_{x} \mathrm{~N}$ with $x=0.028$, these states form bands $\sim 0.2 \mathrm{eV}$ wide.

$\mathrm{Mn}$ atom substituting $\mathrm{Ga}$ in $\mathrm{GaN}$ is nominally a single acceptor. Since the $d(\mathrm{Mn})$ levels are in the gap of GaN, one of the five $d$ electrons fills the hole in the valence band, and the $\mathrm{Mn}$ ion is left in the $d^{4}$ configuration (referred to as $\mathrm{Mn}^{3+}$ ), with two electrons in the $e$ - and two electrons in the $t_{2}$ levels, respectively. Thus, in an intrinsic $(\mathrm{Ga}, \mathrm{Mn}) \mathrm{N}$ sample the Fermi level is located within the $t_{2}$-derived bands at about $1.7 \mathrm{eV}$ above the top of the valence band. The presence of co-dopants and/or defects changes the Fermi energy and thus the charge state of Mn. With changing $E_{\mathrm{F}}, \mathrm{Mn}$ ions may assume charge states that range from $d^{5}$ to $d^{2}$ (i.e., from $\mathrm{Mn}^{2+}$ to $\left.\mathrm{Mn}^{5+}\right)$. In the case of the $d^{5}\left(\mathrm{Mn}^{2+}\right)$ configuration there is one additional electron per $\mathrm{Mn}$ atom and the $t_{2} \uparrow$ state is completely filled. In the opposite case of $d^{2}\left(\mathrm{Mn}^{5+}\right)$, there are two holes per Mn and $t_{2} \uparrow$ is empty.

As follows from Fig. 1, the energies of $d(\mathrm{Mn})$ levels strongly depend on the charge state. For the neutral $\mathrm{Mn}^{3+}$, these levels are at 1.7 and $0.3 \mathrm{eV}$ above the top of the valence band, respectively, which agrees with previous results for both zinc blende $25,26,28$ and wurtzite $22,24,27,29 \mathrm{GaN}$. For the $\mathrm{Mn}^{2+}$ ions, the $t_{2} \uparrow-$ and $e \uparrow$-derived bands are at 2.15 and $0.95 \mathrm{eV}$ above the top of the valence bands, respectively. They are about $0.6 \mathrm{eV}$ higher than for the $\mathrm{Mn}^{3+}$ ions, due to the increased Coulomb repulsion between $d$ electrons. On the other hand, for the $\mathrm{Mn}^{4+}$ and $\mathrm{Mn}^{5+}$ ions with $d^{3}$ and $d^{2}$ configurations, the $e \uparrow$ level is a resonance degenerate with the top of the valence band. We stress that even for the $d^{3}$ configuration the $t_{2}$ level is in the gap, due to the large Coulomb repulsion. Finally, we note that the calculated dependence of the energy levels on the charge state of $\mathrm{Mn}$ is to some extent overestimated due to the self-interaction of $d(\mathrm{Mn})$ electrons. This problem is both relevant and complex, see Ref. 32, but it is outside the scope of this paper.

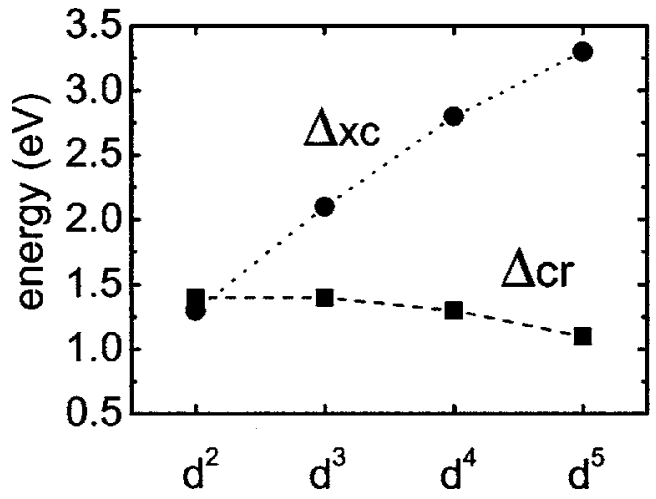

FIG. 2. Calculated crystal field splitting $\Delta_{\mathrm{cr}}$ and exchange splitting $\Delta_{\mathrm{xc}}$ as functions of the charge state of $\mathrm{Mn}$. The values for the $d^{2}$ and $d^{3}$ configurations are approximate, since the $e$ doublet is degenerate with the valence bands.

Spin-up $e \uparrow$ and spin-down $e \downarrow$ states are separated by the exchange energy $\Delta_{\mathrm{xc}}$. As it follows from Fig. 2, the magnitude of $\Delta_{\mathrm{xc}}$ strongly depends on the charge state and increases almost linearly with the number of electrons from about $1.4 \mathrm{eV}$ for the $d^{2}$ configuration to $3.3 \mathrm{eV}$ for the $d^{5}$ one. In the ground state of Mn ion, the $e \downarrow$ level is not occupied with electrons even in $n$-type samples, because it is located about $1 \mathrm{eV}$ above the bottom of the conduction bands for the case of $\mathrm{Mn}^{2+}$. For the other configurations, the $e \downarrow$ level is a deep gap state. This level may be occupied after, e.g., a photon absorption.

The strong dependence on the charge state and a large value of $\Delta_{\mathrm{xc}}$, which is almost two times larger than in GaAs, is due to a couple of factors. In particular, the Coulomb repulsion between the $d$ electrons is stronger than in GaAs due to the more localized character of the $d$ orbitals, which form a state in the $\mathrm{GaN}$ band gap rather than a resonance in the valence bands. Furthermore, the lattice and dielectric constants of $\mathrm{GaN}$ are smaller than in arsenides or phosphides.

We now show that a correct description of the $d(\mathrm{Mn})$ states should include the effects of relaxation of the atoms neighboring Mn. Our calculated lattice constant is $3.14 \AA$ and thus the ideal Ga-N bond length is $1.93 \AA$. For the sequence $\mathrm{Mn}^{2+} \rightarrow \mathrm{Mn}^{5+}$, the calculated equilibrium $\mathrm{Mn}-\mathrm{N}$ bond lengths are $2.01,1.94,1.86$, and $1.79 \AA$, respectively, see Fig. 3. We note that a change of the charge state changes the bond lengths by almost $4 \%$ and that the $\mathrm{Mn}-\mathrm{N}$ bond length is close to the ideal Ga-N value only for the neutral $\mathrm{Mn}^{3+}$. The linear decrease of the bond lengths with the decreasing number of the $d(\mathrm{Mn})$ electrons is due to decreasing Coulomb repulsion with the nearest $\mathrm{N}$ anions. Interestingly, several experimental data ${ }^{16,33-35}$ reveal increased bond lengths of about $4 \%$, when compared with equilibrium value. According to the present results this indicates that the $\mathrm{Mn}$ atoms in these samples are in the $\mathrm{Mn}^{2+}$ charge state. This is possibly due to the presence of nonintentional donors, typical for $\mathrm{GaN}$.

More importantly, the impact of relaxations on the energies of the $d(\mathrm{Mn})$-derived states is substantial and can be neglected only for the neutral $\mathrm{Mn}^{3+}$, for which the relaxation 


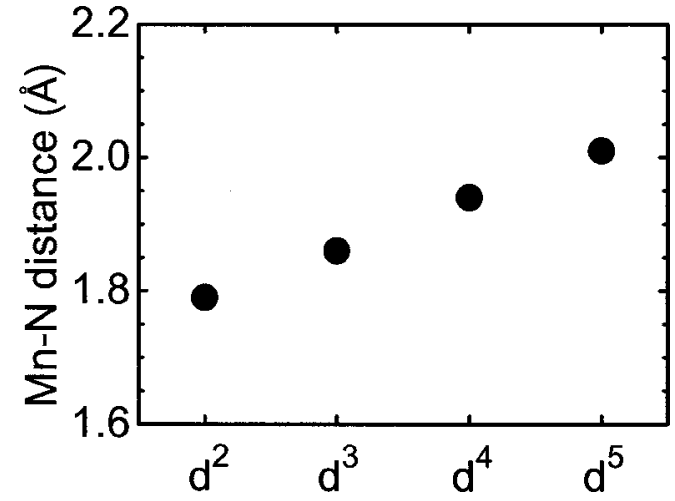

FIG. 3. Dependence of the Mn-N bond length on the charge state of Mn.

is small. In agreement with the antibonding character of the gap states we find that for the $d^{5}$ configuration the outward displacement of the nearest neighbors (NNs) of Mn lowers both the $e$ and the $t_{2}$ states by as much as $0.6 \mathrm{eV}$, while in the case of $d^{3}$ the inward displacement raises the energy of the $t_{2}$ by $0.5 \mathrm{eV} .{ }^{36}$ Finally, the energy gained by displacements of atoms from the unrelaxed to the equilibrium positions is 0.5 , 0.05 , and $0.3 \mathrm{eV}$ for $d^{5}, d^{4}$, and $d^{3}$ cases, respectively. These large values corroborate well with the experimental FrankCondon energies of $0.3 \pm 0.1 \mathrm{eV}$ obtained by Wołos et al. ${ }^{8}$

We have considered the Jahn-Teller (JT) distortions and found that the substitutional locations of both Mn and of its nitrogen neighbors are stable, even with respect to large JT distortions comparable to bond lengths. At equilibrium, Mn is displaced along the $c$ axis from an ideal site, and the symmetry is wurtzite. As was mentioned in Sec. III, at the $\Gamma$ point the Mn-Mn coupling in $\mathrm{Ga}_{0.97} \mathrm{Mn}_{0.03} \mathrm{~N}$ results in splitting and dispersion of the $t_{2}$-derived band into a singlet and a doublet. In general, for $k$ points that are not lying on the high symmetry lines in the Brillouin zone, the doublet is also split, which could lead to a JT effect in the case of the $d^{4}$ charge state. However, the dispersion of the $e$ band is such that for arbitrary $k$ vectors both split bands are occupied with electrons, and JT distortions are not stabilized. This may explain the small value of the JT energy of about $0.035 \mathrm{eV}$ observed in experiment. ${ }^{37}$

\section{MAGNETIC COUPLING}

We now turn to magnetic interactions, which are evaluated by placing two Mn atoms in the unit cell and comparing the energies of the FM and AFM configurations of their spins. According to our results, the position of the Fermi energy determines the sign, the magnitude, and the range of the coupling.

The calculated energy difference $\Delta E^{\mathrm{A}-\mathrm{F}}$ between AFM and FM states allows us to find the coupling $J(r)$, defined by $\Delta E^{\mathrm{A}-\mathrm{F}}=\Sigma J\left(r_{i}\right)$, where the summation runs over all interacting neighbors. Since the coupling mediated by the localized $d(\mathrm{Mn})$-derived gap states is fairly short range, we assume a cut-off radius of $7.5 \AA$, which corresponds to eighthneighbor distance. The potential error in $J$ induced by this assumption should not exceed $2 \mathrm{meV}$. The dependence of

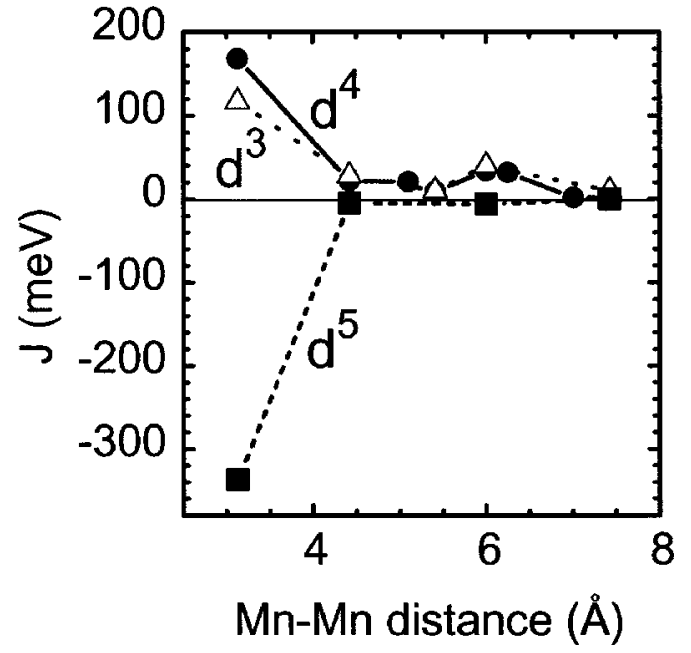

FIG. 4. Calculated coupling as a function of the distance between two $\mathrm{Mn}$ ions which are in $d^{5}$ (squares), $d^{4}$ (circles), and $d^{3}$ (triangles) charge states, and $x=0.056$. Consecutive distances range from the nearest neighbor $\mathrm{Mn}-\mathrm{Mn}$ pair at $3.13 \AA$ to the eighthneighbor pair at $7.42 \AA$. See text.

$J(r)$ on the Mn-Mn separation is shown in Fig. 4. When the Mn ions are in the $d^{5}$ configuration, i.e., when $E_{\mathrm{F}}$ is high due to codoping with donors, the $t_{2}$-derived band is completely filled. In this case we find that the interaction is short-range AFM and strong: The energy difference $\Delta E^{\mathrm{A}-\mathrm{F}}$ between AFM and FM states is $-380 \mathrm{meV}$, see Fig. 4.

In the intrinsic $\mathrm{Ga}_{0.944} \mathrm{Mn}_{0.056} \mathrm{~N}$, all $\mathrm{Mn}$ ions are in the $d^{4}$ state and the $t_{2}$-derived band is $2 / 3$ filled. As Fig. 4 shows, the interaction is FM, but its dependence on the distance is strongly non-monotonic. Finally, when Mn ions are in the $d^{3}$ charge state and the $t_{2}$-derived band is $1 / 3$ filled, the coupling is almost the same as in intrinsic $(\mathrm{Ga}, \mathrm{Mn}) \mathrm{N}$ except for the NN pairs, for which it is almost 50\% lower. This is in contrast to (Ga,Mn)As, where, in agreement with experiment, ${ }^{20}$ the Zener model ${ }^{4}$ predicts a stronger coupling for higher concentrations of holes. The short-range character of the coupling 22,23,24(a),24(c) as well as a change of its sign to $\mathrm{AF}$ in donor-codoped samples were previously predicted within the rigid-band model for varying occupations of the $t_{2}$ band. ${ }^{24(a)}$ The agreement between the results of the rigidband model (which neglects self-consistency and atomic relaxations) with our findings is reasonable.

A simple discussion of the results can rely on the empirical description of magnetism developed in Ref. 38. Within this method, based on the $s p^{3}$ model of the band structure, the Mn-Mn coupling is given by the fourth-order terms in the Mn-band carrier interaction, which involve interband virtual transitions. In the case of the $d^{5}$ configuration, the calculated short range AFM coupling is analogous to superexchange in intrinsic insulating (II,Mn)VI crystal. In the presence of free holes there is a second term of the Rudderman-KittelKasuya-Yosida (RKKY)-type (involving intraband transitions), which may lead to FM coupling. In this case, the interaction is the sum of both contributions. However, $a b$ initio calculations automatically account for the actual band occupation. In this context, the results obtained for the $d^{5}$ case are important because they show that the magnitude of 
the short-range AFM "background" coupling is large. In other words, the results of model calculations for intrinsic $(\mathrm{Ga}, \mathrm{Mn}) \mathrm{As}$ or $(\mathrm{Ga}, \mathrm{Mn}) \mathrm{N}$ may be compared with ab initio results only after adding the short-range AFM contributions.

The effects of atomic relaxations on the NN magnetic interaction are especially large: $\Delta E^{\mathrm{A}-\mathrm{F}}$ increases from 170 to $215 \mathrm{meV}$. For more distant pairs the effect of relaxations is much smaller. This effect is thus comparable to the differences between LSDA and LDA+U schemes. ${ }^{24}$ Therefore, a realistic description of interactions should include both the atomic relaxations and the properly described electronic correlations.

\section{COMPARISON WITH EXPERIMENT}

We now compare our results with experiment. A reasonable agreement for both the electronic and the magnetic properties is obtained for samples with Mn content well below $1 \%$. The scheme of $d(\mathrm{Mn})$-induced levels shown in Fig. 1 is confirmed in particular by

1. Absorption at $\sim 1.42 \mathrm{eV}, 5,6,8$ ascribed to internal transitions between the $e$ and $t_{2}$ states.

2. The direct photoionization of $\mathrm{Mn}$ at about $1.8 \mathrm{eV}$ observed both in photocurrent ${ }^{6}$ and in photo-EPR. ${ }^{8}$

3. Temperature dependence of the photocurrent ${ }^{6}$ showing that the $e$ state is about $0.3 \mathrm{eV}$ above the top of the valence band.

4. EPR investigations of $\mathrm{Mn}^{2+}$ (Refs. 7 and 8) performed in samples with varying $E_{\mathrm{F}}$. Interestingly, within the LDA $+U$ scheme, ${ }^{24(\mathrm{a})}$ the $d(\mathrm{Mn})$ levels are $0.5 \mathrm{eV}$ too low compared with experiment, suggesting that this scheme with a modest value of $U$ of about $4 \mathrm{eV}$ overestimates electron correlation.

Concerning the magnetic coupling, we note that

1. In samples codoped with donors AFM coupling and spin-glasslike magnetization was observed, ${ }^{9,11}$ in accord with our results for the $d^{5}$ configuration.

2. Codoping with $\mathrm{Mg}$ acceptors increases the Curie temperature. ${ }^{16,18}$ The latter effect may occur in samples containing relatively low concentrations of nonintentional donors that are compensated by a comparable amount of $\mathrm{Mg}$. Similarly, FM with $T_{\mathrm{C}}=20 \mathrm{~K}$ observed in Ref. 10 in resistive $(\mathrm{Ga}, \mathrm{Mn}) \mathrm{N}$ is consistent with our results.

Turning to the opposite limit of $(\mathrm{Ga}, \mathrm{Mn}) \mathrm{N}$ with a few percent of Mn we observe that the experimental data are in sharp contradiction with theoretical results. First, both $n$-type ${ }^{12,14,15,17}$ and $p$-type ${ }^{18,19}$ conductivity has been reported, while the $d(\mathrm{Mn})$-derived bands in the middle of the band gap should lead to pinning of $E_{\mathrm{F}}$ and an activated hop- ping conductivity. Second, ferromagnetism persisting above $300 \mathrm{~K}$ (Refs. 10 and 12-18) is in contradiction with theory, which indicates that: (i) magnetic couplings in intrinsic $(\mathrm{Ga}, \mathrm{Mn}) \mathrm{N}$ are weaker than in $(\mathrm{Ga}, \mathrm{Mn}) \mathrm{As}$, where the highest observed $T_{\mathrm{C}}$ is about $170 \mathrm{~K},{ }^{20}$ (ii) in samples with the $\mathrm{Mn}$ content below 1\%, the Mn-Mn distance is large compared to the range of the magnetic coupling, and (iii) in samples codoped with donors, the dominant charge state of $\mathrm{Mn}$ is $d^{5}$ and the predicted coupling is a short-range AFM. There are also experimental results, ${ }^{39,40}$ showing that conduction electrons are weakly coupled to $\mathrm{Mn}$ ions and thus cannot mediate a robust RKKY-type FM.

Since our calculations used a supercell and thus an ordered array of $\mathrm{Mn}$ ions, the effects of random distribution of $\mathrm{Mn}$ on the Curie temperature have been left out. Recently, two groups investigated magnetic percolation effects in $(\mathrm{Ga}, \mathrm{Mn}) \mathrm{N}$ comparing results of the mean-field approximation with the more accurate Monte Carlo solution of the Heisenberg Hamiltonian. ${ }^{41}$ The parameters for the Hamiltonian were extracted from tight-binding linear muffin-tin orbital (LMTO) or Korringa-Kohn-Rustoker (KKR) band structure calculations. While the mean-field approach gives $T_{\mathrm{C}}$ in the experimentally observed range, the more accurate Monte Carlo simulations result in $T_{\mathrm{C}}$ smaller than $100 \mathrm{~K}$ for Mn concentration of $\sim 5 \%$.

\section{CONCLUSIONS}

In summary, we have investigated the electronic structure and magnetic coupling in $(\mathrm{Ga}, \mathrm{Mn}) \mathrm{N}$ alloys by $a b$ initio LSDA calculations, taking into account both the effects of the varying charge state of $\mathrm{Mn}$ and atomic relaxations. Our results satisfactorily explain the available optical and magnetic properties of $(\mathrm{Ga}, \mathrm{Mn}) \mathrm{N}$ in the limit of low $\mathrm{Mn}$ concentrations. In particular, we have demonstrated the importance of lattice relaxations around $\mathrm{Mn}$ ions and of the charge state of Mn, which were previously neglected. ${ }^{22-29}$ However, the observed properties of samples with 1-5\% of $\mathrm{Mn}$ are in drastic contradiction with theoretical results obtained using an ordered structure. While a random distribution of $\mathrm{Mn}$ would result in moderate Curie temperatures, the $400 \mathrm{~K}$ transition temperatures observed in some experiments are indicative of a highly nonhomogeneous distribution of Mn.

\section{ACKNOWLEDGMENTS}

This work was supported by FENIKS project (EC:G5RDCT-2001-00535), Grant No PBZ-KBN-044/P03/2001, and grants from U.S. ONR and DOE. One of us (P.B.) thanks J. Cibert and Z. Wilamowski for helpful discussions.
${ }^{1}$ S. Koshihara, A. Oiwa, M. Hirasawa, S. Katsumoto, Y. Iye, C. Urano, H. Takagi, and H. Munekata, Phys. Rev. Lett. 78, 4617 (1997).

${ }^{2}$ Y. Ohno, D. K. Young, B. Beschoten, F. Matsukura, H. Ohno, and
D. D. Awshalom, Nature (London) 402, 790 (1999).

${ }^{3}$ H. Ohno, D. Chiba, F. Matsukura, T. Omiya, E. Abe, T. Dietl, Y. Ohno, and K. Ohtani, Nature (London) 408, 944 (2000).

${ }^{4}$ T. Dietl, H. Ohno, F. Matsukura, J. Cibert, and D. Ferrand, 
Science 287, 1019 (2000).

${ }^{5}$ R. Y. Korotkov, J. M. Gregie, and B. W. Wessels, Appl. Phys. Lett. 80, 1731 (2002).

${ }^{6}$ T. Graf, M. Gjukic, M. S. Brandt, M. Stutzmann, and O. Ambacher, Appl. Phys. Lett. 81, 5159 (2002).

${ }^{7}$ T. Graf, M. Gjukic, M. Hermann, M. S. Brandt, M. Stutzmann, and O. Ambacher, Phys. Rev. B 67, 165215 (2003).

${ }^{8}$ A. Wołos, M. Palczewska, M. Zając, J. Gosk, M. Kaminska, A. Twardowski, M. Bockowski, I. Grzegory, and S. Porowski, Phys. Rev. B 69, 115210 (2004).

${ }^{9}$ M. Zając, J. Gosk, M. Kaminska, A. Twardowski, T. Szyszko, and S. Podsiadlo, Appl. Phys. Lett. 79, 2432 (2001).

${ }^{10}$ S. Kuwabara, T. Kondo, T. Chikyow, P. Ahmet, and H. Munekata, Jpn. J. Appl. Phys., Part 2 40, L724 (2001).

${ }^{11}$ S. Dhar, O. Brandt, A. Trampert, K. J. Friedland, Y. J. Sun, and K. H. Ploog, Phys. Rev. B 67, 165205 (2003).

${ }^{12}$ G. T. Thaler, M. E. Overberger, B. Gila, R. Frazier, C. R. Abernathy, S. J. Pearton, J. S. Lee, S. Y. Lee, Y. D. Park, Z. G. Khim, J. Kim, and F. Ren, Appl. Phys. Lett. 80, 3964 (2002).

${ }^{13}$ M. L. Reed, N. A. El-Masry, H. H. Stadelmeier, M. K. Ritums, C. A. Parker, J. C. Roberts, and S. M. Bedair, Appl. Phys. Lett. 79, 3473 (2001).

${ }^{14}$ T. Sasaki, S. Sonoda, Y. Yamamoto, K. Suga, S. Shimizu, K. Kindo, and H. Hori, Appl. Phys. Lett. 91, 7911 (2002).

${ }^{15}$ J. Y. Chang, G. H. Kim, J. M. Lee, S. H. Han, H. J. Kim, W. Y. Lee, M. H. Ham, K. S. Huh, and J. M. Muong, J. Appl. Phys. 93, 7858 (2003).

${ }^{16}$ R. Giraud, S. Kuroda, S. Marcet, E. Bellet-Amalric, X. Biquard, B. Barbara, D. Fruchart, D. Ferrand, J. Cibert, and H. Mariette, Europhys. Lett. 65, 553 (2004).

${ }^{17}$ I. T. Yoon, C. S. Park, H. J. Kim, Y. G. Kim, T. W. Kang, M. C. Jeong, M. H. Ham, and J. M. Myoung, J. Appl. Phys. 95, 591 (2004)

${ }^{18}$ K. H. Kim, K. J. Lee, D. J. Kim, H. J. Kim, Y. E. Ihm, C. G. Kim, S. H. Yoo, and C. S. Kim, Appl. Phys. Lett. 82, 4755 (2003).

${ }^{19}$ S. V. Novikov, K. W. Edmonds, A. D. Giddings, K. Y. Wang, C. R. Staddon, R. P. Campion, B. L. Gallagher, and C. T. Foxon, Semicond. Sci. Technol. 19, L13 (2004).

${ }^{20}$ K. W. Edmonds, P. Bogusławski, K. Y. Wang, R. P. Campion, S. V. Novikov, N. R. S. Farley, B. L. Gallagher, C. T. Foxon, M. Sawicki, T. Dietl, M. B. Nardelli, and J. Bernholc, Phys. Rev. Lett. 92, 037201 (2004), and the references therein.

${ }^{21}$ M. Zajac, J. Gosk, E. Grzanka, M. Kaminska, A. Twardowski, B. Strojek, T. Szyszko, and S. Podsiadlo, J. Appl. Phys. 93, 4715 (2003).

${ }^{22}$ B. Sanyal, O. Bengone, and S. Mirbt, Phys. Rev. B 68, 205210 (2003).

${ }^{23}$ P. Mahadevan and A. Zunger, Phys. Rev. B 69, 115211 (2004).

${ }^{24}$ (a) L. M. Sandratskii, P. Bruno, and J. Kudrnovsky, Phys. Rev. B
69, 195203 (2004); (b) A. B. Shick, J. Kudrnovsky, and V. Drchal, ibid. 69, 125207 (2004); (c) J. Kudrnovsky, I. Turek, V. Drchal, F. Maca, P. Weinberger, and P. Bruno, ibid. 69, 115208 (2004).

${ }^{25}$ M. van Schilfgaarde and O. N. Mryasov, Phys. Rev. B 63, 233205 (2001).

${ }^{26}$ U. Gerstmann, A. T. Blumenau, and H. Overhof, Phys. Rev. B 63, 075204 (2001).

${ }^{27}$ E. Kulatov, H. Nakayama, H. Mariette, H. Ohta, and Yu. A. Uspenskii, Phys. Rev. B 66, 045203 (2002).

${ }^{28}$ K. Sato and Hiroshi Katayama-Yoshida, Jpn. J. Appl. Phys., Part 2 40, L485 (2001).

${ }^{29}$ L. Kronik, M. Jain, and J. R. Chelikowsky, Phys. Rev. B 66, 041203 (2002)

${ }^{30}$ S. Baroni, S. de Gironcoli, A. Dal Corso, and P. Gianozzi, http:/ www.pwscf.org

${ }^{31}$ Y. Bar-Yam and J. D. Joannopoulos, Phys. Rev. B 30, 1844 (1984).

${ }^{32}$ A. Filippetti and N. A. Spaldin, Phys. Rev. B 67, 125109 (2003); F. Sokeland, M. Rohlfing, P. Kruger, and J. Pollmann, ibid. 68, 075203 (2003); A. Svane, V. Kanchana, G. Vaitheeswaran, G. Santi, W. M. Tammerman, Z. Szotek, P. Strange, and L. Petit, ibid. 71, 045119 (2005); A. Filippetti, N. A. Spaldin, and S. Sanvito, Chem. Phys. 309, 59 (2005).

${ }^{33}$ Y. L. Soo, G. Kioseglou, S. Kim, S. Huang, Y. H. Kao, S. Kuwabara, S. Owa, T. Kondo, and H. Munekata, Appl. Phys. Lett. 79, 3926 (2001).

${ }^{34}$ M. Sato, H. Tanida, K. Kato, T. Sasaki, Y. Yamamoto, S. Sonoda, S. Shimizu, and H. Hori, Jpn. J. Appl. Phys., Part 1 41, 4513 (2002).

${ }^{35}$ R. Bacewicz, J. Filipowicz, S. Podsiadlo, T. Szyszko, and M. Kaminski, J. Phys. Chem. Solids 64, 1469 (2003).

${ }^{36}$ According to our results, the large energy difference between the $2+/ 3+$ and $3+/ 4+$ levels found by Gerstmann et al. (Ref. 27) is overestimated due to their neglect of relaxations.

${ }^{37}$ A. Wołos, A. Wysmołek, M. Kaminska, A. Twardowski, M. Bockowski, I. Grzegory, S. Porowski, and M. Potemski, Phys. Rev. B 70, 245202 (2004).

${ }^{38}$ B. E. Larson, K. C. Hass, H. Ehrenreich, and A. E. Carlsson, Phys. Rev. B 37, 4137 (1988).

${ }^{39}$ A. Wołos, M. Palczewska, Z. Wilamowski, M. Kaminska, A. Twardowski, M. Bockowski, I. Grzegory, and S. Porowski, Appl. Phys. Lett. 84, 5328 (2004).

${ }^{40}$ K. Ando, Appl. Phys. Lett. 82, 100 (2003).

${ }^{41}$ L. Bergqvist, O. Eriksson, J. Kudrnovsky, V. Drchal, P. Korzhavyi, and I. Turek, Phys. Rev. Lett. 93, 137202 (2004); K. Sato, W. Schweika, P. H. Dederichs, and H. Katayama-Yoshida, Phys. Rev. B 70, 201202(R) (2004). 